\title{
A Novel Cylindrical DRA for C-Band Applications
}

\author{
Hamed Gharsallah, Lotfi Osman and Lassaad Latrach \\ Department of Physics \\ UR "CSEHF" 13ES37 \\ Faculty of Sciences of Tunis, University of Tunis El Manar, 2092 Tunisia
}

\begin{abstract}
In this paper, we study a Dielectric Resonator Antenna of cylindrical shape with circular polarization for applications in the $\mathbf{C}$ band. The proposed antenna is composed of two different layers. The first is Polyflon Polyguide with relative permittivity $\varepsilon_{\mathrm{r} 1}=\mathbf{2 . 3 2}$ and a loss tangent $\tan \delta=0.002$ as a lower layer. The second is Rogers $\mathrm{RO3010}$ with relative permittivity $\varepsilon_{\mathrm{r} 2}$ $=10.2$ and a loss tangent $\tan \delta=\mathbf{0 . 0 0 3 5}$ as an upper layer which is excited by dual probe feed. The $90^{\circ}$ phase shift of two probes feed can create a circular polarization. In this study, we focused on the effect of the variations in the height of the Polyflon Polyguide as well as the probe feed. Simulations under HFSS software have led to bandwidth values of about $2.2 \mathrm{GHz}$ and $2.6 \mathrm{GHz}$ for the proposed antenna with one probe and dual probe, respectively. The obtained gains are higher than $5.4 \mathrm{~dB}$ and can range up 8.1 dB.
\end{abstract}

Keywords-Dielectric Resonator Antenna; gain; reflection coefficient; circular polarization; axial ratio

\section{INTRODUCTION}

Nowadays, many researchers are interested in the dielectric resonators. This theme is introduced in 1983 [1], and widely studied and used over the last two decades owing to their attractive properties like for examples the small size, the simplicity of feeding by classical methods and the very high radiation efficiency compared to microstrip antennas [2]. The Dielectric Resonator Antenna (DRA) has characteristics that can be modified according to an appropriate choice of dimensions of the resonator as well as its dielectric constant.

Let us note that the production of dielectric resonators (DR) don't add additional constraints and can also easily adjust the quality factor and the resonant frequency [3]-[4]. A cylindrical DRA is simple to design allowing an easy control of the resonant frequency and the Q-factor by the ratio radius/height and the permittivity $\varepsilon_{r}$. It would be necessary to know that it is possible to excite the different modes of this type of DRA by adjusting the position and the type of excitation, thereby obtaining an omnidirectional or broadside radiation pattern [5].

The circular polarization (CP) is preferred in the antenna applications because this system gives a wide flexibility in the orientation of the antennas in transmission mode as well as in reception mode [6], and can increase the bandwidth [7]-[8]. The broadband CP antenna applications include many areas such as radar, RFID and satellite. In satellite applications, a $\mathrm{CP}$ is preferred in order to overmaster the consequences of the rotation of polarization due to the atmosphere. In the case of radar applications, the $\mathrm{CP}$ signals are used so as to obtain a maximum of information about the target [9].
The $\mathrm{CP}$ is a combined excitation of two degenerate orthogonal modes. The vector of the electric describes a circle over time and a helical motion along the propagation direction. The DRA is used for many reasons among which the very broad bandwidth. For a single DRA, the use of a cylindrical or a conical shape can increase this bandwidth. A dielectric constant of low value can reduce the quality factor $\mathrm{Q}$ and thereafter increases the width of the bandwidth [10]. In order to obtain best results, we will focus on another technique using a multilayer configuration for different permittivities $\varepsilon_{r}$ [11]-[12].

In this paper, a cylindrical dual layer dielectric resonator circularly polarized antenna excited by a dual coaxial probe feed is proposed for C-band applications. To achieve a wideband performance, we need to stack two dielectric layers one over the other. Therefore, the lower layer is of type Polyflon Polyguide with relative permittivity $\varepsilon_{\mathrm{r} 1}=2.32$ and a loss tangent $\tan \delta=0.002$, and the upper layer is of type Rogers RO3010 of dielectric constant $\varepsilon_{\mathrm{r} 2}=10.2$ and a loss tangent $\tan \delta=0.0035$. To make sure to get good impedance matching and ensure at the same time wideband performance, it is necessary to use a coaxial probe feed excitation. It should also be known that a dual coaxial probe feed inclined by $90^{\circ}$ can create the circular polarization.

The best advantage of the use of a coaxial probe feed excitation is the direct coupling into a $50-\Omega$ system without the need of a matching network [13]. The effects of the probe and the Polyflon Polyguide heights will be studied through the use of the HFSS commercial software [14].

We present in this paper the simulation results of the return loss, gain and axial ratio of the cylindrical dielectric resonator antenna (CDRA) that we propose with dual layer and that works in the C-band.

\section{ANTENNA DESIGN}

Fig.1 shows the geometry of the proposed cylindrical dielectric resonator antenna. The shape of the DRA, the type and the location of the excitation are responsible of the antenna characteristics.

The cylindrical shape of the dielectric resonator gives a large degree of freedom. Thereby, the coaxial probe feed located adjacent to a CDRA offers a large coupling and thereafter a high gain and a symmetric radiation pattern. The mode excited with this location is the $H E M_{11 \delta}$ mode. 


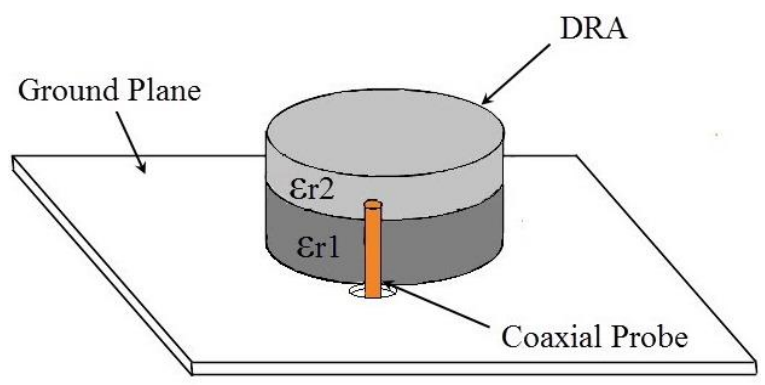

(a)
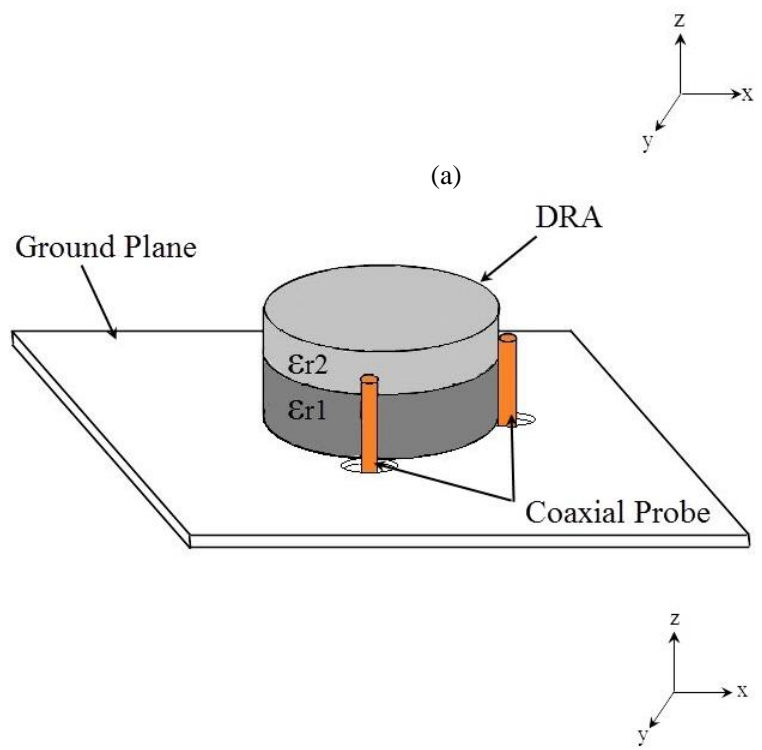

(b)

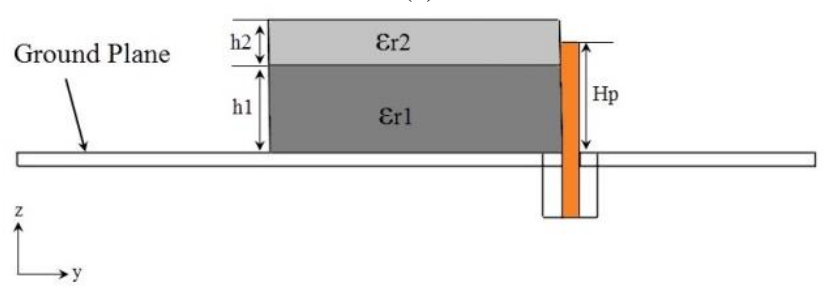

(c)

Fig. 1. Cylindrical DRA design, (a) with only one probe feed, (b) with two probes feed, (c) cut-view (y-z plane)

The relation (1) gives the formula of the resonance frequency of a CDRA corresponding to the case of $\mathrm{HEM}_{11 \delta}$ mode [15].

$$
f=\frac{6.324 c}{2 \pi \sqrt{2+\varepsilon_{\mathrm{r}}}}\left\{0.27+0.36 \frac{\mathrm{a}}{2 \mathrm{~h}}+0.02\left(\frac{\mathrm{a}}{2 \mathrm{~h}}\right)^{2}\right\}
$$

where $\varepsilon_{\mathrm{r}}$ is the permittivity, $a$ is the radius and $\mathrm{h}$ is the height of the antenna. Its quality factor is expressed by the following relation (2).

$$
\mathrm{Q}=0.01007\left(\varepsilon_{\mathrm{r}}\right)^{1.3}\left(\frac{\mathrm{a}}{\mathrm{h}}\right)\left[1+100 \mathrm{e}^{-\left[2.05\left(\frac{\mathrm{a}}{2 \mathrm{~h}}\right) \frac{1}{\left.80\left(\frac{\mathrm{a}}{\mathrm{h}}\right)^{2}\right]}\right]}\right.
$$

The percentage bandwidth is given by

$$
\% \mathrm{BW}=\frac{\Delta \mathrm{f}}{\mathrm{f}_{\mathrm{r}}} * 100=\frac{\mathrm{s}-1}{\mathrm{Q} \sqrt{\mathrm{s}}} * 100
$$

where $\Delta \mathrm{f}$ and $\mathrm{f}_{\mathrm{r}}$ are the bandwidth and the resonant frequency, respectively. The VSWR is the voltage standing wave ratio of the antenna.

Equation (4) shows the relation between $\mathrm{h}$ and $\varepsilon_{\mathrm{r}}$.

$$
\varepsilon_{\mathrm{reff}}=\frac{\mathrm{h}_{\mathrm{eff}}}{\mathrm{h}_{1} / \varepsilon_{\mathrm{r} 1}+\mathrm{h}_{2} / \varepsilon_{\mathrm{r} 2}} \text { with } \mathrm{h}_{\mathrm{eff}}=\mathrm{h}_{1}+\mathrm{h}_{2}
$$

where $h_{1}$ and $h_{2}$ are the heights of Rogers RO3010 (upper layer) and Polyflon Polyguide (lower layer), respectively.

This equation indicates the influence of the couple thickness-permittivity on the resonance frequency and the Qfactor, and thereby the bandwidth for the dual layer dielectric resonator antenna [14]. The parameters $\varepsilon_{\text {reff }}$ and $h_{\text {eff }}$ replace $\varepsilon_{\mathrm{r}}$ and $\mathrm{h}$ in (1) and (2) so that $\varepsilon_{\text {reff }}$ becomes the effective permittivity and $h_{\text {eff }}$ the effective height. Fig. 1(a) and Fig. 1(b) exhibit the 3-D view of the dual layer cylindrical dielectric resonator antenna with one probe feed and dual probe feed, respectively. Fig. 1 (c) represents the cut-view (y$\mathrm{Z}$ plane) of this structure. It is composed of a Polyflon Polyguide characterized by $\varepsilon_{\mathrm{r} 1}=2.32$ as lower layer and a Rogers RO3010 with $\varepsilon_{\mathrm{r} 2}=10.2$ as upper layer. The proposed antenna is located in the middle of the ground plane with dimensions equal to $\mathrm{L}_{\mathrm{g}} \times \mathrm{W}_{\mathrm{g}} \times \mathrm{H}_{\mathrm{g}}$. The radii of the lower and upper layers of the antenna are identical but with different heights.

The use of a coaxial probe has no effect on the matching of the antenna and the circular polarization is generated from the $90^{\circ}$ phase shift. The optimized value of the height $\mathrm{Hp}$ is 8.2 mm thus obtaining a good reflection coefficient at the resonant frequency.

Table I below shows the optimized parameters of the proposed antenna.

TABLE I. OPTIMIZED PARAMETERS OF THE PROPOSED ANTENNA

\begin{tabular}{|l|l|}
\hline Dimension & Value (mm) \\
\hline $\mathrm{L}_{\mathrm{g}}$ & 60 \\
\hline $\mathrm{H}_{\mathrm{g}}$ & 1 \\
\hline $\mathrm{h}_{1}$ & 6.67 \\
\hline $\mathrm{H}_{\mathrm{p}}$ & 8.2 \\
\hline $\mathrm{W}_{\mathrm{g}}$ & 60 \\
\hline $\mathrm{a}$ & 11 \\
\hline $\mathrm{h}_{2}$ & 3.33 \\
\hline
\end{tabular}

\section{RESULTS AND DISCUSSION}

\section{A. Parametric Results}

The results are obtained for the reflection coefficient versus frequency. We achieved a wide bandwidth for the antenna double layer where the top layer has a higher permittivity and the inferior layer has a lower permittivity. The upper layer is made of Rogers RO3010 with $\varepsilon_{\mathrm{r} 2}=10.2$, whereas the lower layer is made of Polyflon Polyguide with $\varepsilon_{\mathrm{r} 1}=2.32$.

The simulation results of the S11 parameter for different values of h1 and Hp are shown in Fig. 2. We can confirm that an interesting impedance bandwidth of about $38 \%$ is obtained in the $[4.6-6.8] \mathrm{GHz}$ frequency range for both $\mathrm{h}_{1}=6.67 \mathrm{~mm}$ (Fig. 2a) than for $\mathrm{Hp}=8.2 \mathrm{~mm}$ (Fig. 2b). 


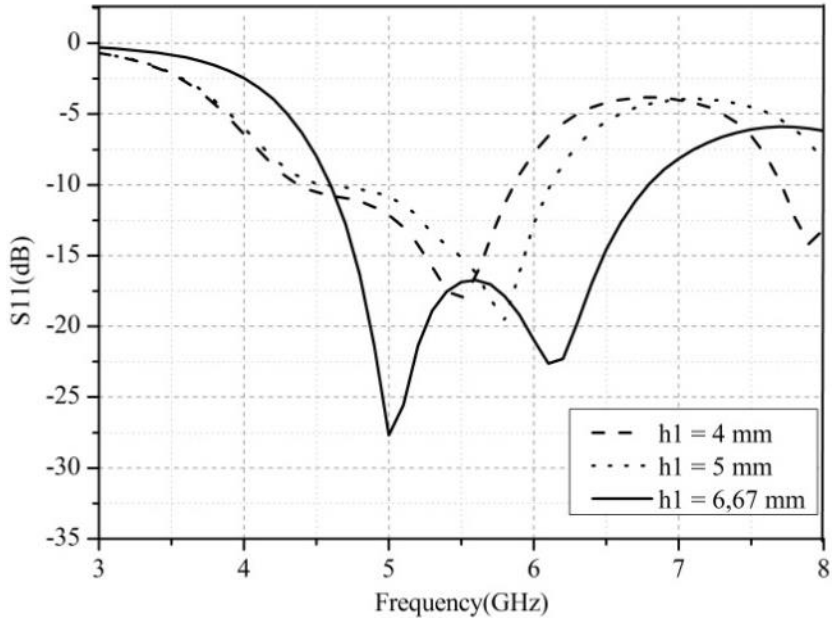

(a)

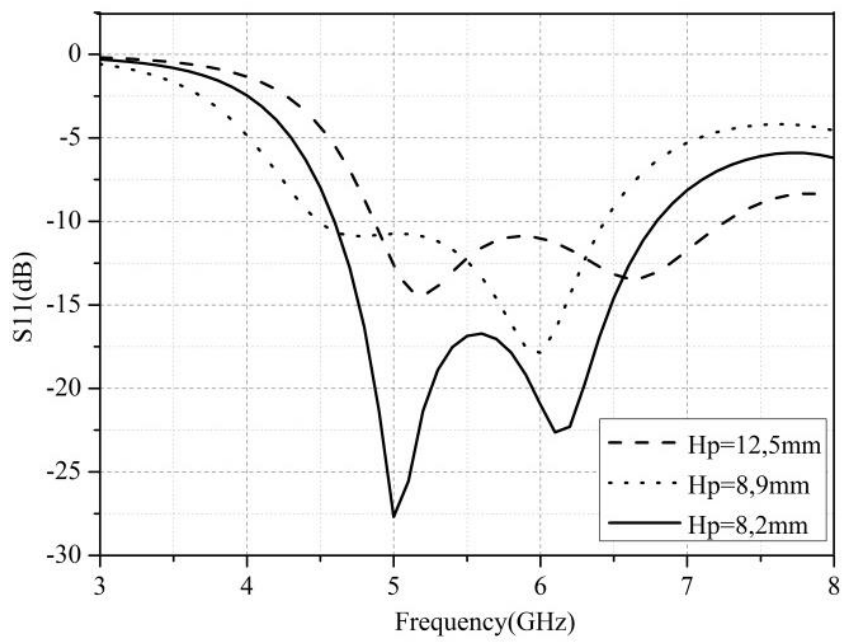

(b)

Fig. 2. Reflection coefficient of the proposed dual segment cylindrical DRA with one probe feed: (a) for different values of $h_{1}$, (b) for different values of $\mathrm{Hp}$

Fig. 3 shows the simulation result of the S11 parameter in the case of two probes feed shifted $90^{\circ}$ degree. It is this inclination that creates the circular polarization.

We notice that the impedance bandwidth is around $44 \%$ in the frequency band [4.5-7.1] GHz. Therefore, we can deduce that the circular polarization is able to increase the bandwidth and that the latter is larger in the case of dual probe feed than in that of only one probe feed.

This increase of the bandwidth can range from $37.9 \%$ up to $44 \%$ in the [4.5-7.2] GHz frequency band.

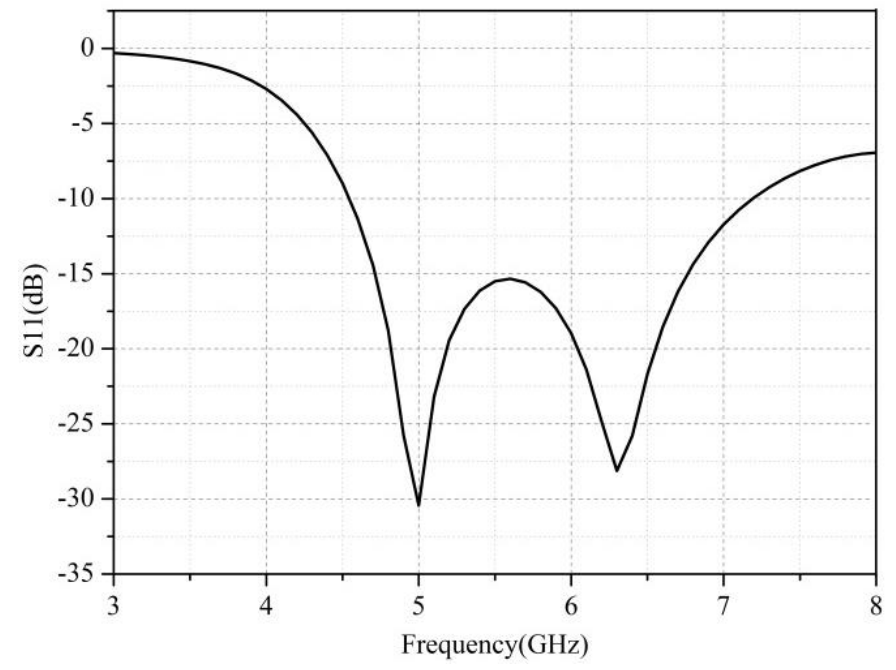

Fig. 3. Reflection coefficient of the proposed dual layer cylindrical DRA with two probes feed

Fig. 4 shows a comparison of the simulation results of the S11 parameter carried out under Ansoft HFSS and CST studio softwares.

The difference observed between the two curves is due to different calculation methods of these two commercial tools.

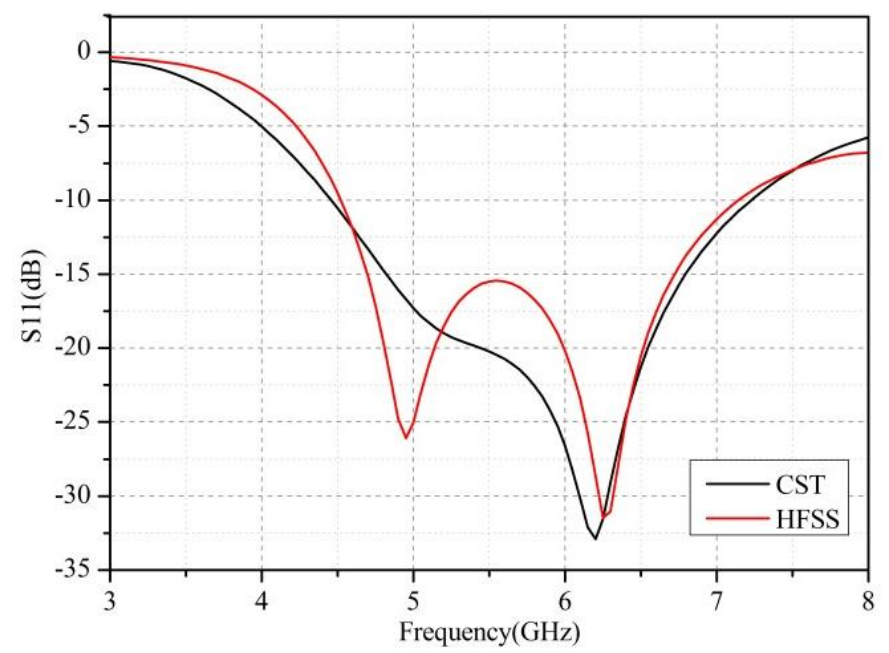

Fig. 4. Comparison of the S11 results performed with Ansoft HFSS and CST studio sotwares

B. Axial Ratio

Fig. 5 exhibits the result of the simulated axial ratio of the proposed antenna with two probes feed. It generates the circular polarization in all the bandwidth. 


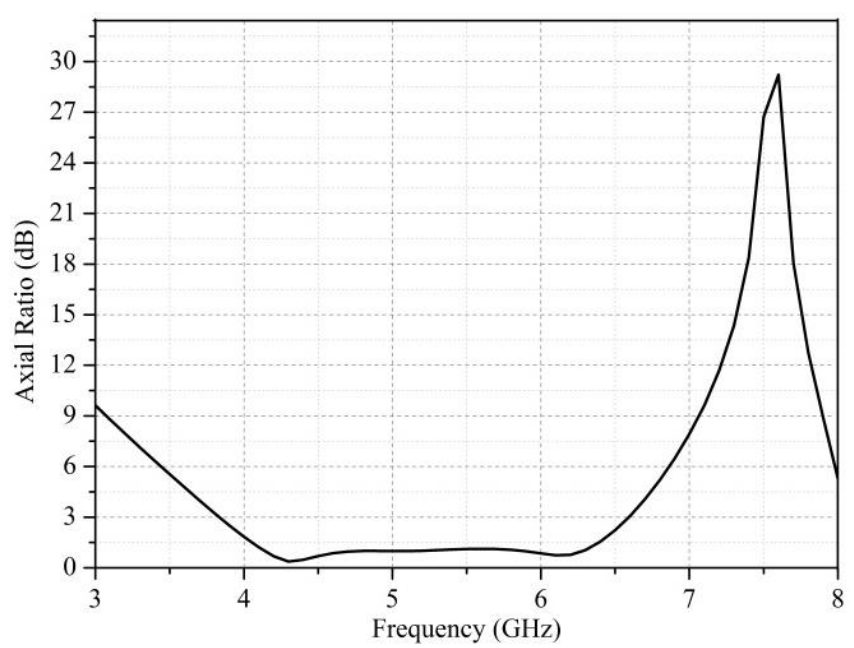

Fig. 5. Simulated axial ratio of the proposed dual layers cylindrical DRA with dual probe feed

\section{Gain}

Fig. 6 shows the simulation results of the gain over a frequency range from $4.5 \mathrm{GHz}$ to $7.1 \mathrm{GHz}$.

The proposed dual layer cylindrical DRA with two probes feed offers simulated values of gain reaching $8.1 \mathrm{~dB}$ and no less than $5.4 \mathrm{~dB}$ in the frequency range from $4.5 \mathrm{GHz}$ to 7.1 $\mathrm{GHz}$.

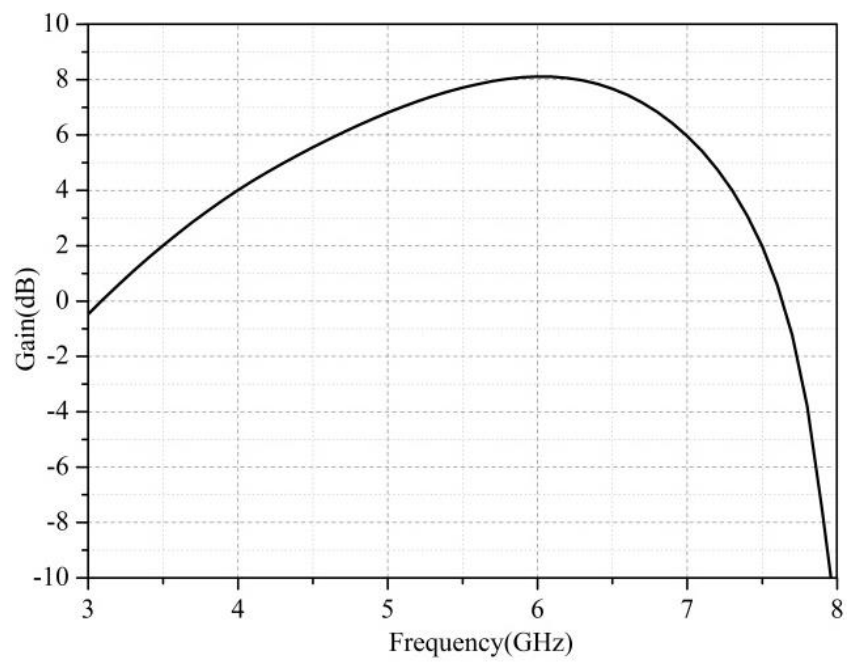

Fig. 6. Gain of the proposed dual layer cylindrical DRA with dual probe feed

Fig. 7 shows that the simulated gain in $3 \mathrm{D}$ at $5 \mathrm{GHz}, 5.8$ $\mathrm{GHz}$ and $6.4 \mathrm{GHz}$ frequencies are $7 \mathrm{~dB}, 8 \mathrm{~dB}$ and $7.9 \mathrm{~dB}$, respectively. We denote that the antenna gain is constant throughout the bandwidth.

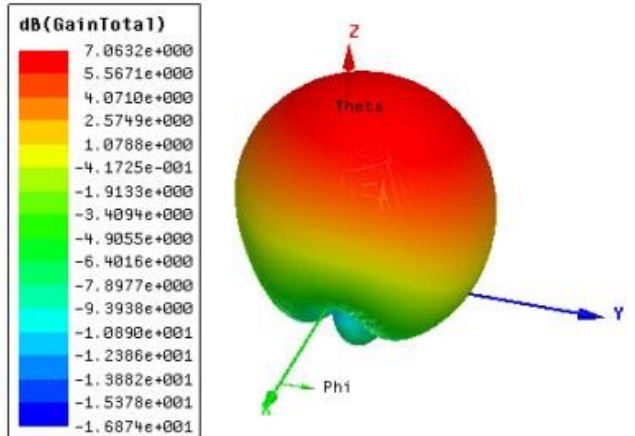

(a)

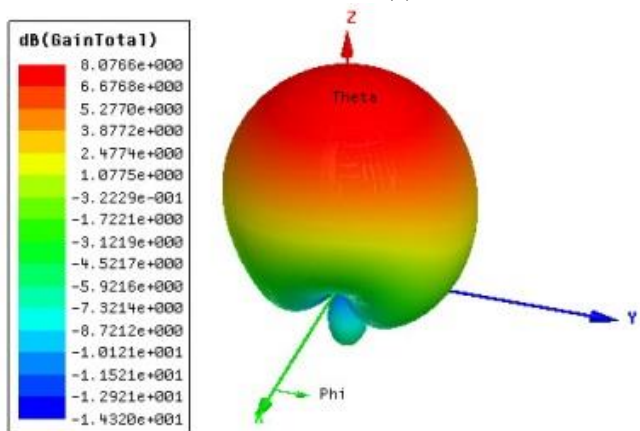

(b)

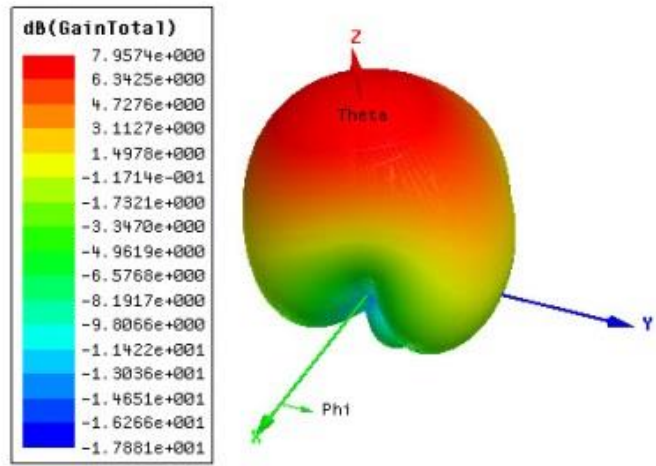

(c)

Fig. 7. Gain of the proposed antenna: (a) $5 \mathrm{GHz}$, (b) $5.8 \mathrm{GHz}$ and (c) 6.4 $\mathrm{GHz}$

\section{Radiation Patterns}

Fig. 8 shows the polar representation of the radiation patterns obtained in the E-plane ( $\mathrm{x}-\mathrm{z}$ plane) and the H-plane ( $\mathrm{y}-\mathrm{z}$ plane), respectively with $\mathrm{phi}=0^{\circ}$ and $\mathrm{phi}=90^{\circ}$ at the resonant frequency of $5.8 \mathrm{GHz}$. The radiation pattern is broadside in the two planes. 


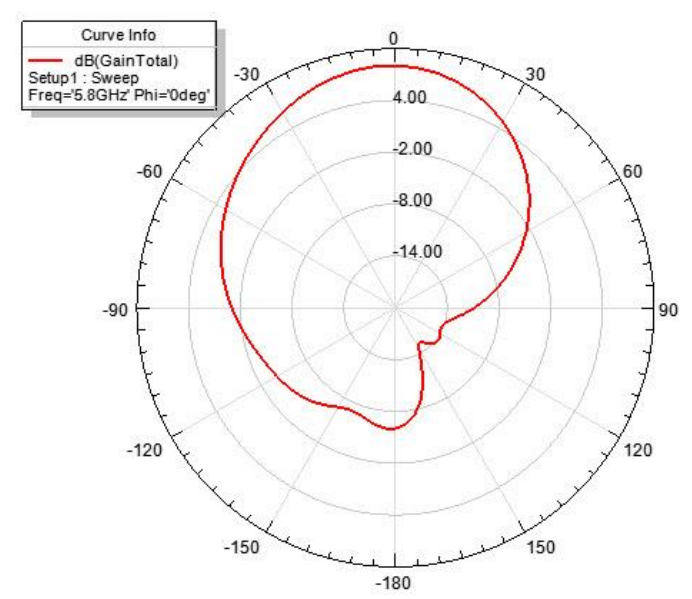

(a)

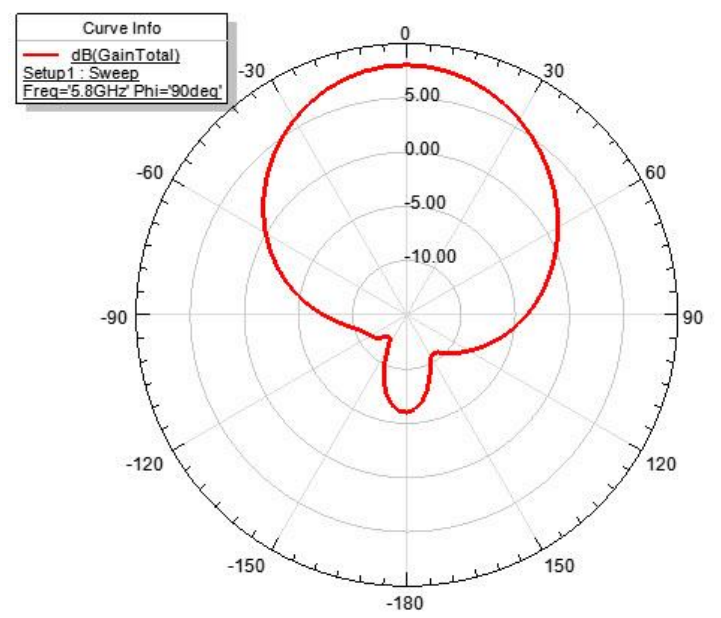

(b)

Fig. 8. Radiation patterns of the antenna at $5.8 \mathrm{GHz}$. (a) in the E-Plane (x-y plane), (b) in the H-plane (y-z plane)

\section{CONCLUSION}

A dual layer cylindrical dielectric resonator antenna with circular polarization functioning in C-band has been studied in this paper. The obtained results of this proposed antenna showed an increase of the bandwidth of up to $44 \%$ in the [4.57.2] GHz frequency band with two probes feed, and the gain is able to reach $8.1 \mathrm{~dB}$ in this frequency band. Finally, we remind that this antenna is designed for WLAN/WIMAX communication systems operating in the C-band.

\section{REFERENCES}

[1] S. A. Long, M.W. McAllister, and L. C. Shen, "The resonant cylindrical dielectric cavity antenna," IEEE Transactions on Antennas and Propagation, Vol. 43, AP-31, No.3, May 1983.

[2] Petosa Aldo, Dielectric Resonator Antennas Handbook, Artech House, 2007.

[3] A. A. Kishk and A. W. Glisson, "Bandwidth Enhancement For Split Cylindrical Dielectric Resonator Antennas," Progress In Electromagnetics Research, PIER 33, 97-118, 2001.

[4] H. Y. Huang, J. Y. Wu, and K. L. Wong, "Cross-Slot-Coupled Microstrip Antenna and Dielectric Resonator Antenna for Circular Polarization," IEEE Transactions on Antennas and Propagation, Vol. 47, No. 4, April 1999.

[5] A. W. Glisson, D. Kajfez, and J. James, "Evaluation of modes in dielectric resonators using a surface integral equation formulation," IEEE Trans. Microwave Theory Tech., vol. ATT-31, no.12, December 1983.

[6] L. C. Y. Chu, D. Guha, and Y.M.M. Ontario, "Comb-shaped circularly polarized dielectric resonator antenna," Electronics Letters, Vol. 42 No. 14, 6th July 2006.

[7] H. Gharsallah, L. Latrach, and L. Osman, "Development of a Novel Wideband Conical Dielectric Resonator Antenna," IEEE 15th Mediterranean Microwave Symposium (MMS), Lecce, Italy, Nov. 30 2015-Dec. 22015.

[8] Y. D. Zhou, Y. C. Jiao, Z. B. Weng, and T. Ni, "A Novel Single-Fed Wide Dual-Band Circularly Polarized Dielectric Resonator Antenna," IEEE Antennas and Wireless Propagation, Vol. 15, 2016.

[9] R. Y. Sun, "Bandwidth Enhancement of Circularly Polarized Dielectric Resonator Antenna," ETRI Journal, Volume 37, Number 1, February 2015.

[10] R. K. Chaudhary, K. V. Srivastava, and A. Biswas, "Three-Element Multilayer Multipermittivity Cylindrical Dielectric Resonator Antenna For Wideband Applications With Omnidirectional Radiation Pattern And Low Cross-Polarization," Microwave and Optical Technology Letters, Vol. 54, No. 9, September 2012.

[11] Y. Geand and K. P. Esselle, "A Dielectric Resonator Antenna for UWB Applications," IEEE Transactions on Antennas and Propagation, 2009.

[12] R. K. Chaudhary, K. V. Srivastava, and A. Biswas, "Four Element Multilayer Cylindrical Dielectric Resonator Antenna Excited by a Coaxial Probe for Wideband Applications," Communications (NCC), National Conference on Bangalore, 2011.

[13] Z. N. Chen., et al., "Effect of Parasitic Disk on a Coaxial Probe-Fed Dielectric Resonator Antenna," Microwave and Optical Technology Letters," Vol. 15, No. 3, June 1997, pp. 166-168.

[14] "Ansoft Corporation", High Frequency Structure Simulator, v 15.

[15] A. Rashidian, K. Forooraghi, and M. R. Tayefeh, "A New Method For Calculating The Resonant Frequency of Multisegment Dielectric Resonator Antennas," Microwave \& Telecommunication Technology, 2003. 